\title{
DYNAMICS OF ACOUSTIC PROPAGATION THROUGH A SOLITON WAVE PACKET: OBSERVATIONS FROM THE INTIMATE'96 EXPERIMENT
}

\author{
O.C. RODRIGUEZ AND S.M. JESUS \\ UCEH-Universidade do Algarve (UALG) \\ Campus de Gambelas, \\ Faro, Portugal \\ Y. STEPHAN AND X. DEMOULIN \\ Service Hydrographique et Océanographique \\ de la Marine (SHOM) \\ Brest, France \\ M.B. PORTER \\ New Jersey Institute of Technology (NJIT) \\ Newark, USA \\ AND \\ E.F. COELHO \\ Instituto Hidrográfico (IH) \\ Lisbon, Portugal
}

\begin{abstract}
.
Experimental observations of acoustic propagation through a Soliton Wave Packet (SWP) show an abnormally large attenuation over some frequencies, that was found to be significantly time dependent and anisotropic. Nevertheless, by remarking the problem of signal attenuation, the approach used in most of the studies can be considered as "static" since no additional effects were taken into account as a SWP evolves in range and time. Hydrographic and acoustic data from the INTIMATE'96 experiment clearly exhibit traces of the presence of soliton packets, but in contrast with known observations of attenuation its frequency response also reveals a sudden increase of signal amplitude, which may be due to a focussing effect. This signal increase appears to be in coincidence with a significant peak found in current and temperature records. However, the correlation of both acoustic and hydrographic features is difficult to support due to the different
\end{abstract}




\section{O.C. RODRÍGUEZ ET AL.}

time scales between the rate of hydrographic data sampling and the rate of signal transmissions. In order to study the possibility that a SWP could be responsible for the observed signal increase the INTIMATE'96 hydrographic data was used to generate physically consistent distributions of "soliton-like" fields of temperature and sound velocity, which were used as input for a range-dependent normal-mode model; it was found that for a particular soliton field the set of "dynamic" (i.e., range-dependent and time-dependent) acoustic simulations reveals an acoustic signature similar to that observed in the data. These results contribute to a better understanding of underwater propagation in shallow-water coastal environments and therefore provide a potential basis for range-dependent temperature and sound speed inversions.

\section{Introduction}

It is known that naturally generated solitons can be often observed in coastal zones, as a result of nonlinear interaction of the surface tide with the continental shelf; the generation mechanism remains however poorly understood. The significant circulation of organic surfactants caused by SWPs induces small displacements of the ocean level and leads to a modulation of the sea-surface roughness which can be clearly detected by satellite SAR images. Such surface signatures provide a detailed information about the propagation characteristics of SWPs (Small et al., 1995). Soliton packets have been observed by satellite almost everywhere in coastal zones and in particular near Portugal. Observations of propagating solitons include also a considerable amount of current and temperature measurements. For instance during the summer of 1994 current and temperature data taken near Porto (Sherwin et al., 1996) allowed for the observation of a wave packet, constituted by three solitons, which could be tracked during its propagation towards the shore. The waves were characterized by sudden isotherm depressions of up to $45 \mathrm{~m}$ lasting 10-35 minutes, accompanied by current surges of up to $0.45 \mathrm{~m} / \mathrm{s}$ and shears of up to $0.7 \mathrm{~m} / \mathrm{s}$ (over $60 \mathrm{~m}$ ). SWPs propagated away from the shelf break towards the shore with an average speed of $0.56 \mathrm{~m} / \mathrm{s}$ and appeared each tidal cycle, which confirms the important role of tides as a significant source for the generation of SWPs.

The problem of acoustic propagation through SWPs has been intensively studied in the last years, essentially in order to explain the anomalous frequency response of shallow-water propagating signals, which were found to be strongly time dependent, anisotropic and sometimes exhibiting an abnormally large attenuation over some frequency range (Zhou et al., 1991), (Caille et al., 1997). Most of the known reports remarked the problem of signal attenuation, without regarding to additional effects as a soliton packet evolves in time and range. Hydrographic and acoustic data 
from the INTIMATE'96 experiment clearly exhibit traces of soliton presence. However, in contrast with referenced observations of attenuation the frequency response reveals also a "soliton-like" acoustic signature which corresponds to an increase of signal amplitude. Such acoustic feature can be due to a focussing effect. In order to study the possibility that the signal increase could be caused by the presence of a SWP in the acoustic waveguide the hydrographic data was used to generate physically consistent distributions of "soliton-like" fields of temperature and sound velocity, which were used as input for a range-dependent acoustic propagation model. As will be shown in the following sections the results of acoustic "dynamic" (i.e., range-dependent and time-dependent) simulations strongly support the assumption that a particular SWP was responsible for the observed soliton-like acoustic signature.

\section{Theoretical Background}

\subsection{HYDRODYNAMIC NORMAL MODES}

For an environment with complex stratification the main characteristics of SWPs depend on the Hydrodynamic Normal Modes (hereafter HNMs), which are solutions of the linear rotationless form of coupled Hydrodynamic Equations. The dependence of SWPS on HNMs is of central importance for a detailed description of soliton propagation. For this reason this theoretical background will start with a short introduction regarding HNMs.

In the absence of rotation the system of linear coupled Hydrodynamic Equations for the dynamic fields of current, density and pressure can be written as follows (Apel, 1987):

$$
\begin{gathered}
\frac{\partial u}{\partial x}+\frac{\partial v}{\partial y}+\frac{\partial w}{\partial z}=0, \\
\frac{\partial \rho}{\partial t}+\frac{d \rho_{0}}{d z} w=\frac{\partial \rho}{\partial t}-N^{2} \frac{\rho_{0}}{g} w=0, \\
\frac{\partial u}{\partial t}=-\frac{1}{\rho_{0}} \frac{\partial p}{\partial x} \quad, \quad \frac{\partial v}{\partial t}=-\frac{1}{\rho_{0}} \frac{\partial p}{\partial y}, \\
0=-\frac{\partial p}{\partial z}-\rho g .
\end{gathered}
$$

It can be shown that the dynamic fields can be expanded in terms of HNMs $\Psi_{m}$ and $\phi_{m}$ in the following way:

$$
\begin{gathered}
w=\sum_{m} w_{m} \Psi_{m} \quad, \quad(u, v)=D \sum_{m}\left(u_{m}, v_{m}\right) \phi_{m}, \\
\rho=\frac{\rho_{0} N^{2}}{g} \sum_{m} \rho_{m} \Psi_{m} \quad, \quad p=\rho_{0} \sum_{m} p_{m} \phi_{m},
\end{gathered}
$$


where $N^{2}$ is the buoyancy frequency, which can be properly estimated from the mean temperature $T_{0}(z), D$ is the water depth, $\phi_{m}=d \Psi_{m} / d z$ and $\Psi_{m}$ obeys the following differential equation:

$$
\frac{d^{2} \Psi_{m}}{d z^{2}}+\frac{N^{2}}{C_{m}^{2}} \Psi_{m}=0
$$

Under top and bottom boundary conditions $\Psi_{m}(0)=\Psi_{m}(D)=0$ the HNMs will constitute two different orthogonal sets:

$$
\left\langle\phi_{m} \phi_{n}\right\rangle=\left\langle N^{2} \Psi_{m} \Psi_{n}\right\rangle=0
$$

where $m \neq n$ and the "inner product" \langle\rangle is defined as

$$
\langle\ldots\rangle=\int_{0}^{D} \ldots d z .
$$

\subsection{THE KORTEWEG-DE VRIES "MODAL" EQUATION}

Let us consider now that $p=p_{0}+p^{\prime}, \rho=\rho_{0}+\rho^{\prime}$, with $d p_{0} / d z+\rho_{0} g=0$, where $\left(\rho^{\prime}, p^{\prime}\right)$ are perturbations from a mean state $\left(\rho_{0}(z), p_{0}(z)\right)$. Under this assumption the nonlinear rotationless form of coupled Hydrodynamic Equations can be written as follows:

$$
\begin{gathered}
\nabla_{h} \vec{U}_{h}+\frac{\partial w}{\partial z}=0, \\
\frac{\partial \rho^{\prime}}{\partial t}+\frac{d \rho_{0}}{d z} w=-w \frac{\partial \rho^{\prime}}{\partial z}, \\
\rho_{0} \frac{\partial \vec{U}_{h}}{\partial t}+\nabla_{h} p^{\prime}=-\left[\rho^{\prime} \frac{\partial \vec{U}_{h}}{\partial t}+\rho_{0} w \frac{\partial \vec{U}_{h}}{\partial z}+\rho_{0}\left(\vec{U}_{h} \nabla_{h}\right) \vec{U}_{h}\right], \\
\frac{\partial p^{\prime}}{\partial z}+\rho^{\prime} g=-\rho_{0} \frac{\partial w}{\partial t},
\end{gathered}
$$

where $\vec{U}_{h}=\vec{i} u+\vec{j} v$ and $\nabla_{h}=\vec{i} \partial / \partial x+\vec{j} \partial / \partial y$. This time the nonlinear fields of current, density and pressure can be expanded as

$$
\begin{gathered}
\vec{U}_{h}=D \sum_{m} \overrightarrow{\mathcal{U}}_{m} \phi_{m}, \rho^{\prime}=N^{2} \sum_{m} C_{2 m} \rho_{m} \Psi_{m}, \\
p^{\prime}=\rho_{0} \sum_{m} C_{3 m} p_{m} \phi_{m},
\end{gathered}
$$




$$
w=\frac{\partial \xi}{\partial t}+\nabla_{h}\left(\vec{U}_{h} \xi\right), \xi=\sum_{m} \eta_{m} \Psi_{m}
$$

where $\xi$ represents nonlinear vertical displacement. Using the orthogonal properties of HNMs and neglecting modal coupling and highly nonlinear terms the set of nonlinear coupled equations can be reduced to the set of Boussinesq equations (Ostrovsky, 1978), (Gabov, 1988):

$$
\begin{gathered}
\frac{\partial \eta_{m}}{\partial t}+D \nabla_{h} \overrightarrow{\mathcal{U}}_{m}+\frac{1}{2} s \nabla_{h}\left(\overrightarrow{\mathcal{U}}_{m} \eta_{m}\right)=0 \\
\frac{\partial \overrightarrow{\mathcal{U}}_{m}}{\partial t}+\frac{C_{m}^{2}}{D} \nabla_{h} \eta_{m}+s\left(\overrightarrow{\mathcal{U}}_{m} \nabla_{h}\right) \overrightarrow{\mathcal{U}}_{m}-\frac{s}{2 D} \frac{\partial}{\partial t}\left(\eta_{m} \overrightarrow{\mathcal{U}}_{m}\right)+d D \nabla_{h} \frac{\partial^{2} \eta_{m}}{\partial t^{2}}=0,
\end{gathered}
$$

where $s=D\left\langle\phi_{m}^{3}\right\rangle /\left\langle\phi_{m}^{2}\right\rangle$ is a dimensionless parameter of modal nonlinearity and $d=D^{-2}\left\langle\Psi_{m}^{2}\right\rangle /\left\langle\phi_{m}^{2}\right\rangle$. Considering that the nonlinear perturbation propagates along the $x$ axis and by taking (Gabov, 1988)

$$
\mathcal{U}_{m}=\frac{C_{m}}{D} \eta_{m}+\frac{1}{4} s \frac{C_{m}}{D^{2}} \eta_{m}^{2}+\frac{1}{2} d D C_{m} \frac{\partial^{2} \eta_{m}}{\partial x^{2}}
$$

and $\partial / \partial t \approx-C_{m} \partial / \partial x$ it can be obtained that modal vertical displacement $\eta_{m}$ obeys the following nonlinear equation:

$$
\frac{\partial \eta_{m}}{\partial t}+C_{m} \frac{\partial \eta_{m}}{\partial x}+\frac{3}{2} s \frac{C_{m}}{D} \eta_{m} \frac{\partial \eta_{m}}{\partial x}+\frac{1}{2} d D^{2} C_{m} \frac{\partial^{3} \eta_{m}}{\partial x^{3}}=0
$$

which is called the Korteweg-de Vries "Modal" equation (hereafter KdV). The KdV equation applies also to $\mathcal{U}_{m}$, from which follows that $\mathcal{U}_{m} \sim \eta_{m}$. This implies that in the case of soliton propagation both modal vertical displacement and current modal amplitudes will exhibit similar "solitonlike" shapes. In contrast with the corresponding equation for a homogeneous fluid (Gabov, 1988), which admits the generation of a single soliton, it follows from Eq.(1) that each HNM generates a "modal" soliton, with characteristics that depend on that HNM.

\subsection{THE "SECH" SOLUTION}

For displacements having large enough amplitudes and steepness it can be shown that the KdV equation admits the well-known "sech" solution (Apel et al., 1997):

$$
\eta_{m}=\eta_{m}^{0} \operatorname{sech}^{2}\left(\frac{x-\mathcal{C}_{m} t}{\Delta_{m}}\right)
$$


where $\eta_{m}^{0}$ represents the peak amplitude of the modal soliton, which has a nonlinear characteristic width

$$
\Delta_{m}=\sqrt{\frac{12 \beta_{m}}{\alpha_{m} \eta_{m}^{0}}},
$$

and propagates with a nonlinear phase speed given by

$$
\mathcal{C}_{m}=C_{m}+\frac{\alpha_{m} \eta_{m}^{0}}{3}
$$

As seen from the above equations $\Delta_{m}$ is inversely proportional to the amplitude of the modal soliton, whereas $\mathcal{C}_{m}$ is linearly proportional to $\eta_{m}^{0}$. The implication is that the larger $\eta_{m}^{0}$, the faster the soliton propagates and the narrower or steeper the soliton is. The solution given by Eq.(2) describes a single nonlinear perturbation, which propagates in both time and range without deforming its shape. In this way a single modal "sech" solution does not agree with observations, which show the propagation of SWPs exhibiting dispersive properties and made up of different "components". However, a reasonable explanation for this is that each component of the SWP corresponds to a particular "sech" profile and dispersion is a direct consequence of the different phase speeds of packet components.

\subsection{THE "DNOIDAL" SOLUTION}

Another solution to the KdV equation is (Apel et al., 1997):

$$
\eta_{m}=\eta_{m}^{0}\left[2 \operatorname{dn}_{(m, s)}^{2}\left(\frac{x-\mathcal{C}_{m} t}{\Delta_{m}}\right)-\left(1-\mathrm{s}^{2}\right)\right]
$$

where the index $\mathrm{S}$ is a complex function of normalized variable $\tau=x / C_{m} t$ and $\operatorname{dn}_{s}(\varphi)$ is the "dnoidal" Jacobi elliptic function. Shapes of the "dnoidal" solution agree well with backscattered profiles measured from SAR images (Apel et al., 1997). As $S \rightarrow 1$ the above expression becomes the "sech" profile. The dynamics of a "dnoidal" soliton is completely different than the one of the "sech" profile. Eq.(4) describes not a single but an entire SWP which evolves in time and range. The number of solitons within the packet depends on $\mathrm{S}$ and $\tau$. This implies that one can derive entire sets of "dnoidal" soliton packets from a single HNM.

For certain parameter choices the "dnoidal" profile resembles better the observations of SWPs. However, this leads to some ambiguity because if a single "dnoidal" solution resembles an entire packet it is not clear which of the modal solutions has to be considered, and there is also the possibility that the packet is made up of several "dnoidal" components. This matter is 
clearly related with the discussion of packet propagation in terms of "sech" components and will be recalled during the discussion of thermistor data.

\subsection{TEMPERATURE PERTURBATIONS}

From the analysis of coupled nonlinear and rotationless form of Hydrodynamic Equations it follows that modal amplitudes of horizontal current components depend linearly on modal vertical displacement $\left(\mathcal{U}_{m}, \mathcal{V}_{m}\right) \sim$ $\eta_{m}$. Nevertheless, from a tomographical point of view, the system of Hydrodynamic Equations does not provide a physical basis for expanding the sound speed field. To address the tomographic issue let us recall the thermodynamical equation (LeBlond et al., 1989)

$$
\frac{D}{D t}\left(\rho C_{v} T\right)=\nabla\left(k_{T} \nabla T\right)+Q_{T}
$$

where $C_{v}$ denotes the specific heat at constant volume, $k_{T}$ is the thermal conductivity and $Q_{T}$ represents all sources and sinks of heat. Linearizing and solving this differential equation (Rodríguez et al., 1998) one can obtain that:

$$
T \approx T_{0}(z)+\frac{d T_{0}}{d z} \sum_{m} T_{m} \Psi_{m}
$$

where $T_{m} \sim \eta_{m}$. The approximation becomes linear when $d T_{0} / d z \approx$ constant.

\section{Hydrographic and Acoustic Data}

The INTIMATE'96 experiment, performed during the summer 1996, North of Lisbon (see Fig.1), was the first experiment on underwater acoustics to be performed in Portuguese waters and involved the collaboration of several institutions: the Universidade do Algarve, Faro, the Instituto Hidrográfico, Lisbon, both from Portugal and the Centre Militaire OceanographiqueSHOM, Brest, France, and has also received support from the Saclant Undersea Research Centre, La Spezia, Italy. The experiment was conceived with the main goal of applying the methods of Ocean Acoustic Tomography to the detection and inversion of the internal tide. The area of the experimental site was chosen because of the potential presence of internal tides and internal waves. Some characteristics of the experimental site were known from previous surveys performed by the Instituto Hidrográfico.

The general strategy of the INTIMATE'96 experiment was the following (see Fig.2): the French vessel BO'DENTRECASTEAUX carried the acoustic source which emitted a 2 seconds long LFM chirp with a bandwidth of $500 \mathrm{~Hz}$ between 300 and $800 \mathrm{~Hz}$, repeated every 8 seconds. The 


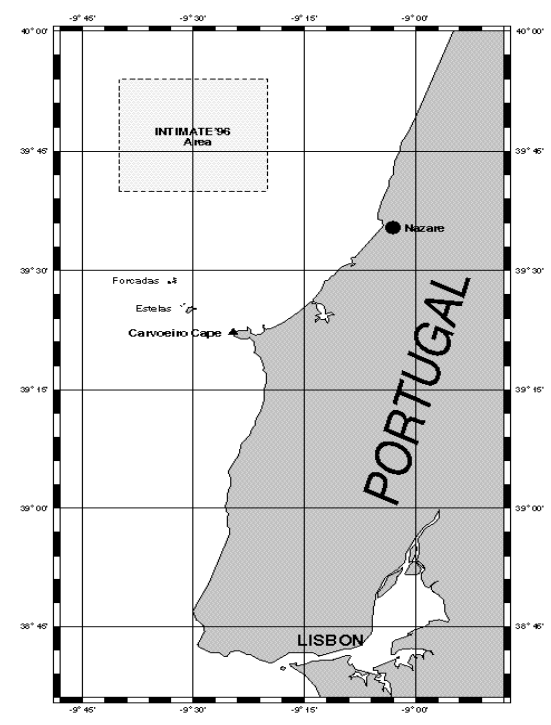

Figure 1. The INTIMATE'96 experimental site

Real Data Acquisition Scenario

INTIMATE'96, JUN 1996 - NW Nazaré site

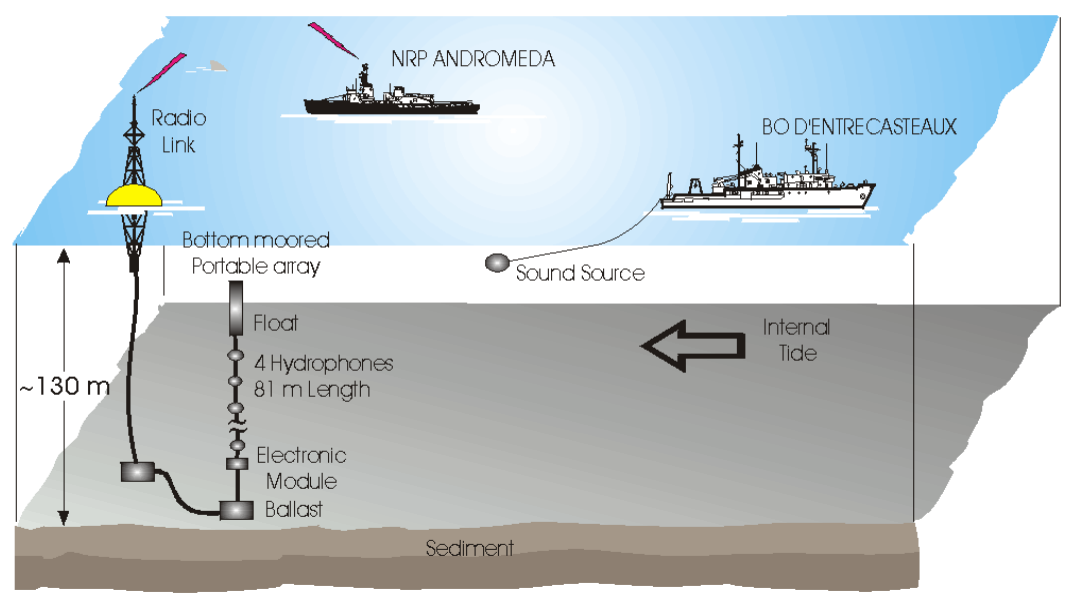

Figure 2. General strategy of the INTIMATE'96 experiment 


\section{DYNAMICS OF ACOUSTIC PROPAGATION THROUGH A SWP}

signal was received on a Vertical Linear Array (hereafter VLA) with four hydrophones, and then transmitted by radio to the Portuguese vessel NRP ANDROMEDA, for online monitoring and backup.

Transmissions were performed from North and West positions, along range-independent and range-dependent acoustic tracks, respectively, with corresponding distances of 5.6 and $6.4 \mathrm{kms}$ (see Fig.3). During the experiment it was also conducted an intensive survey of thermistor, CTD, XBT and ADCP data, which allowed for the calculations of empirical orthogonal functions (hereafter EOFs) of currents and temperature, and also for the calculations of HNMs (see Fig.4). In particular a high degree of correlation was found between EOFs and HNMs, up to mode 3 (Rodríguez et al., 1998). This is very significant since it indicates that every HNM is equivalent to the corresponding EOF. Furthermore, the quantity and resolution of EOFs depends on the number and resolution of measured profiles, while HNMs can be obtained from a coarse estimate of mean temperature and still provide a detailed description of the environmental dynamics. Once the HNMs were determined, the relationship Eq.(3) was used to calculate the characteristic soliton width $\Delta_{m}$ as a function of peak amplitude $\eta_{m}^{0}$ (see Fig.5). An important result of these calculations is that $\Delta_{m}$ is complex except for HNMs 1, 5, 11, 15 and 19. This simplifies significantly the analysis of propagating SWPs since together with the degree of correlation between HNMs and EOFs mentioned previously, the ambiguity related to the structure of soliton packets is eliminated: only the first modal solution of the $\mathrm{KdV}$ equation will be responsible for the generation of SWPs. Whether the packet corresponds to a "sech" profile or a "dnoidal" profile depends on the particular conditions of soliton generation, which is beyond the scope of this study.

Following the same type of analysis presented in (Apel et al., 1997), and taking advantage of the correlation between EOFs and HNMs, the empirical "modal" amplitudes of hydrographic data were filtered into low-pass and high-pass frequency components; the cutoff frequency for separation was chosen in order to obtain a "tidal" band (with periods shorter than 18h) and a "buoyancy" band (with longer periods). Due to the low sampling frequency $(\sim 1 / 10$ minutes $)$ the estimation of vertical displacement from thermistor data did not resolve the structure of soliton packets. Nevertheless it was expected to "capture" some of the solitons within a packet -if anyin the high-pass frequency component; they could be recognized as being part of a SWP due to the correlation of such peaks with the maximae of the low-pass frequency component. However, the processing of data did not provide any physical information about the direction and phase velocity of SWPs due to the lack of information at other locations.

The results of filtering are shown in Figs.6 and 7. There are two common 
O.C. RODRÍGUEZ ET AL.

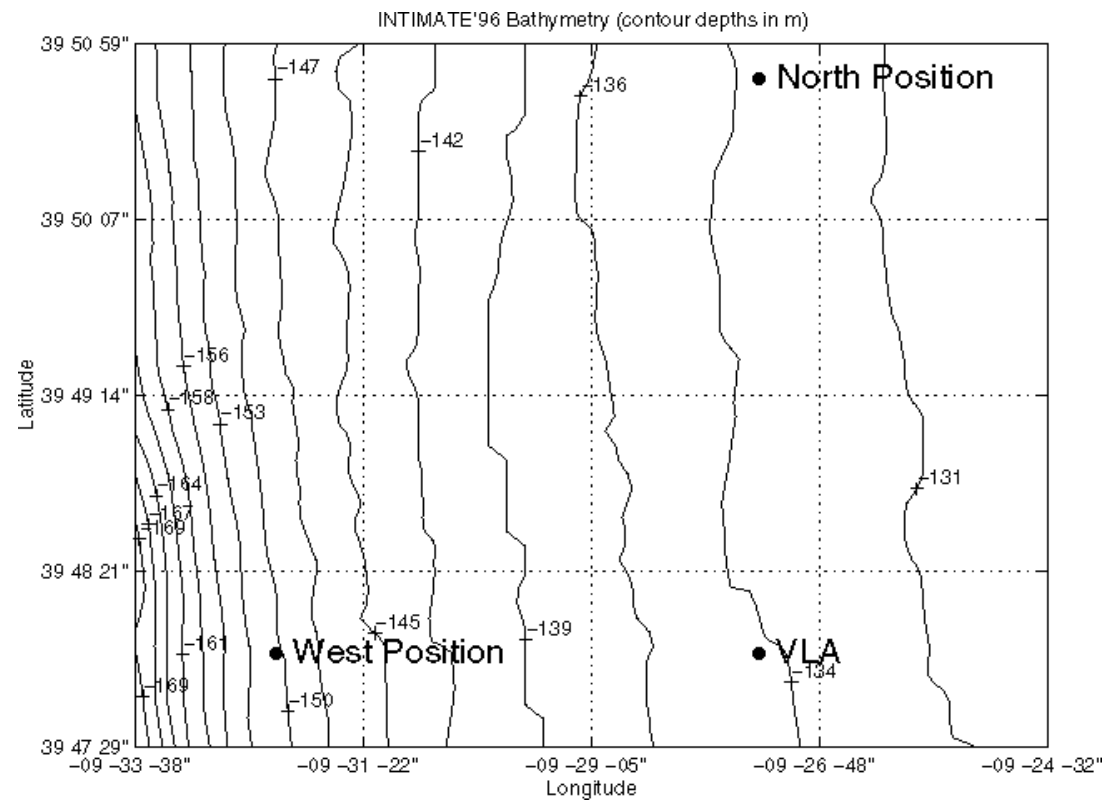

Figure 3. General bathymetry of the INTIMATE'96 experiment.

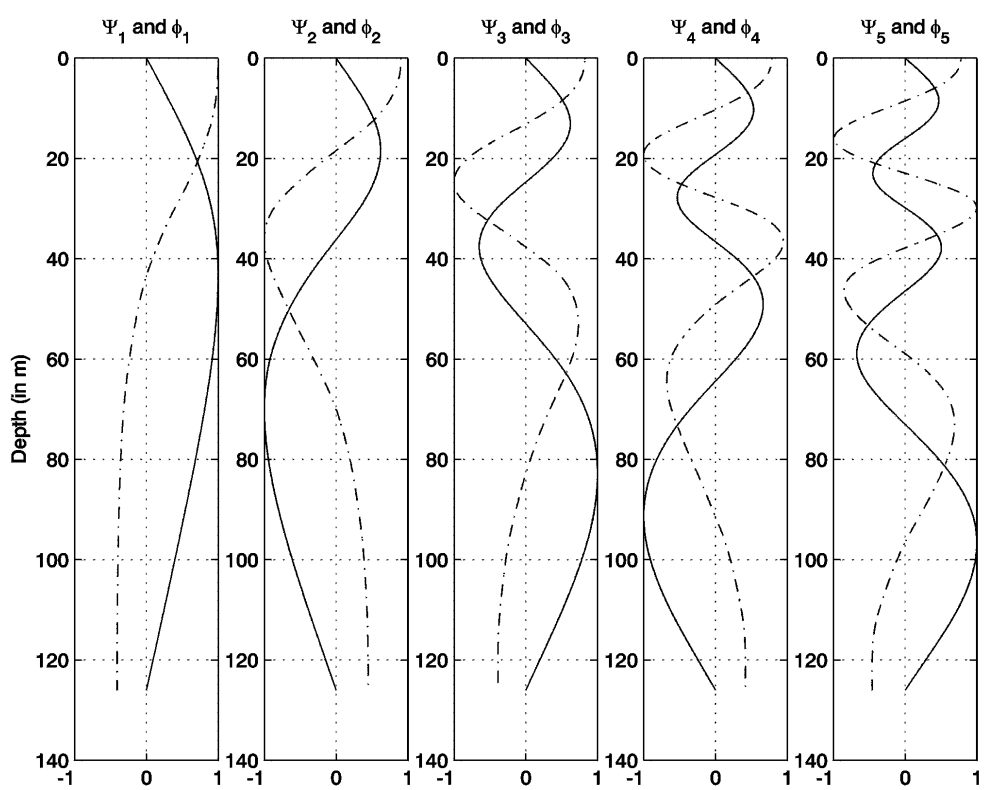

Figure 4. Normalized Hydrodynamic Normal Modes $\Psi_{m}$ (continuous line) and their derivatives $\phi_{m}$ (dott-dashed line) calculated from CTD data near the VLA. 


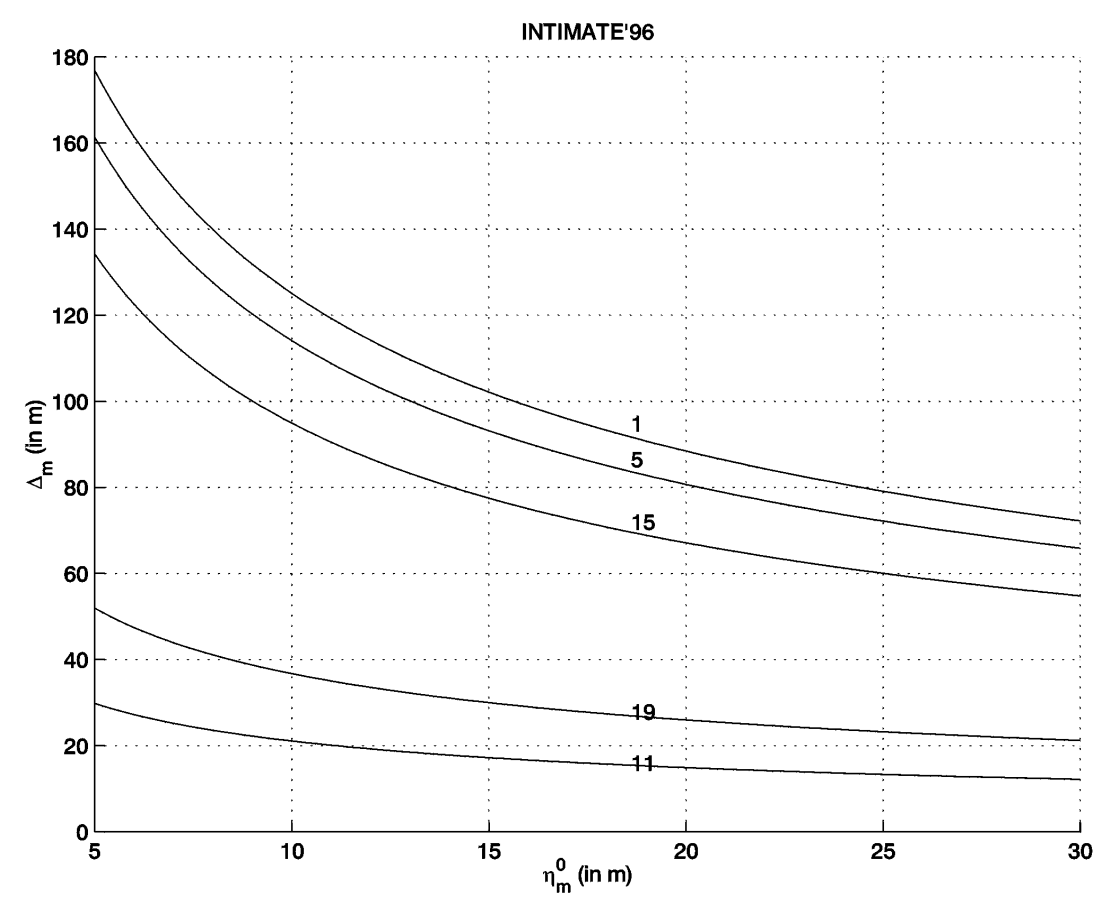

Figure 5. Characteristic modal soliton widths $\Delta_{m}$ as a function of their peak amplitude $\eta_{m}^{0}$, with numbers indicated the corresponding indexes of the Hydrodynamic Normal Mode.

features that can be seen in both figures: the first is the tidal oscillation of the low-frequency components, which is related to the process of propagation of the internal tide; the second is the presence of significant "peaks" in all high-frequency components. For the case of currents (see Fig.6) peaks reach amplitudes up to $40 \mathrm{~cm} / \mathrm{s}$, which agrees with observations from (Apel et al., 1997) and (Sherwin et al., 1996). The distribution of peaks is not arbitrary. By looking at their positions (see for instance Fig.7) it becomes clear that peaks are "aligned" with the maximae of the low-frequency component, indicating propagation of tidal solitons. Peaks are located slightly behind the maximae. A reasonable explanation for this is that the phase speeds of the internal tide and SWPs are different, leading to a difference in travel times as the internal tide and the SWPs propagate away from the shelf break towards the shore.

\section{Acoustic Data and Simulations}

From an acoustic point of view the third peak found in the high pass filtered thermistor data of Fig. 7 was of particular interest, since part of acoustic 
O.C. RODRÍGUEZ ET AL.
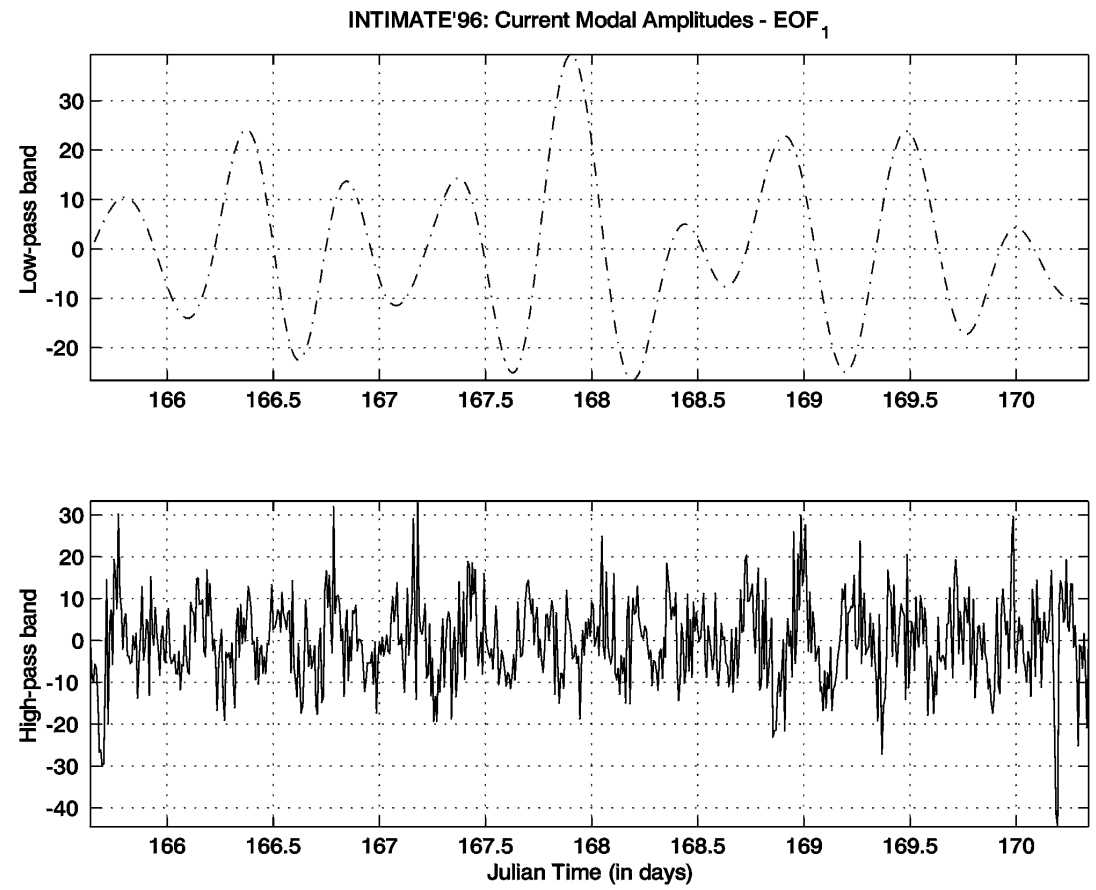

INTIMATE' 96: Current Modal Amplitudes - EOF
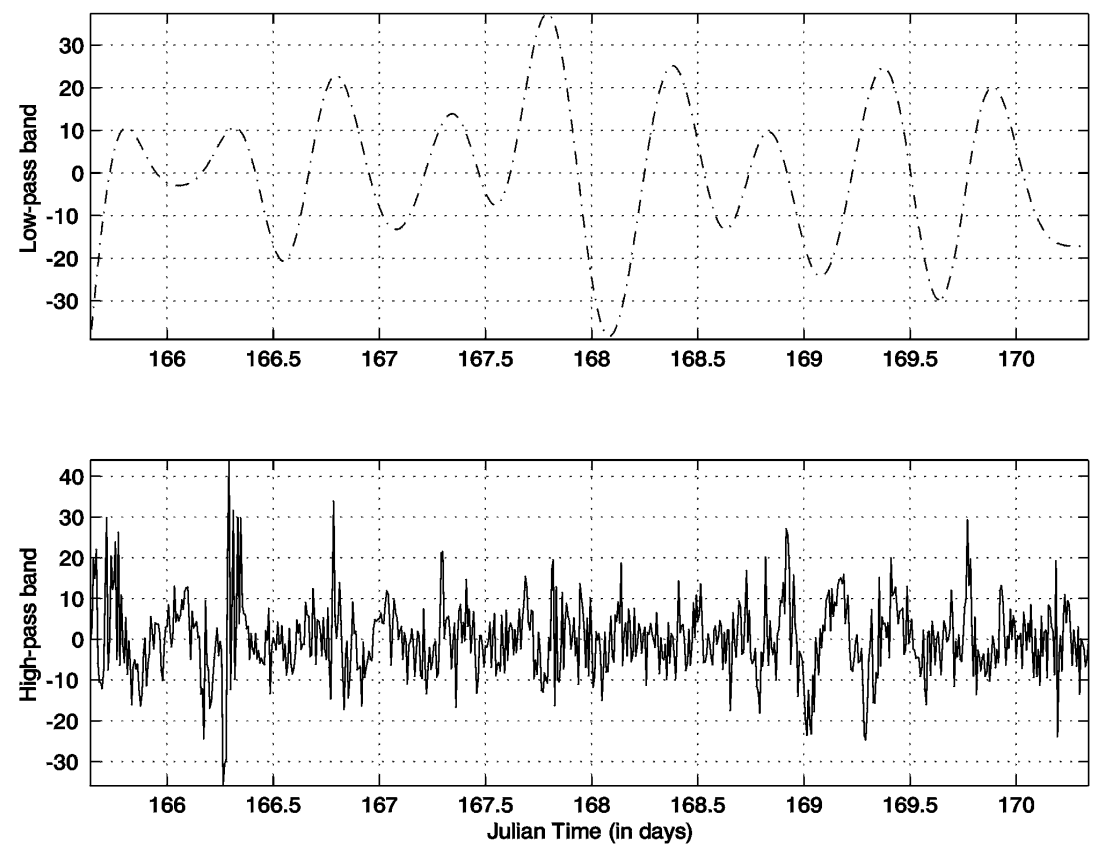

Figure 6. Filtered modal amplitudes of horizontal current components $u$ (top) and $v$ (bottom); units are given in $\mathrm{cm} / \mathrm{s}$. 

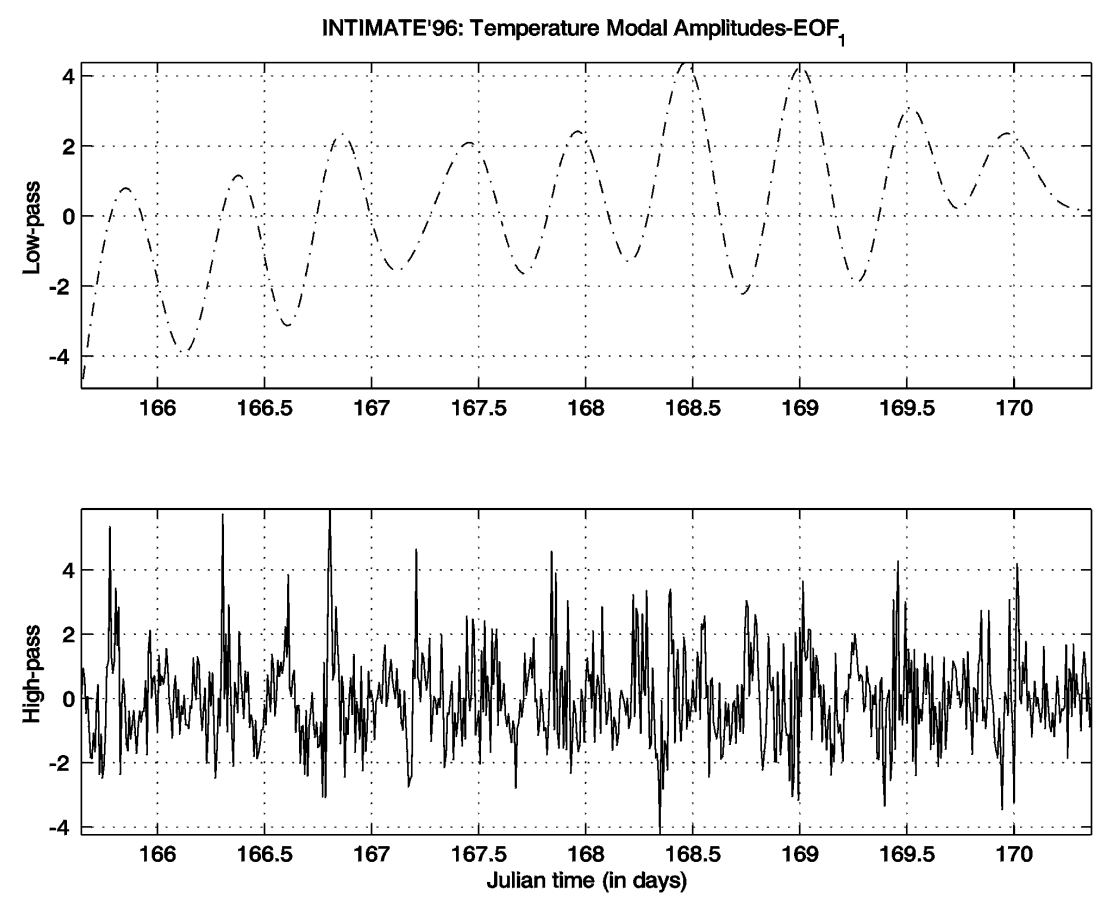

Figure 7. Filtered modal amplitude of temperature; units are given in Celsius degrees.

transmissions covered a temporal window of 3 hours around that peak. The corresponding calculation of relative Transmission Loss (see Fig.8) revealed two bright symmetric "stripes" for which TL increases along frequencies. This is an effect compatible with that expected from acoustic propagation through a SWP. However, the most interesting feature is a "soliton-like" signature between the "stripes". It corresponds to an increase of signal amplitude lasting over 15 minutes and can be due to a sort of focussing effect. Such particular behaviour of TL along time is not described in any of the consulted referencies. The increase in signal is significantly enhanced in the curve of TL at $430 \mathrm{~Hz}$ (see Fig.9). Unfortunately the correlation between the considered peak of thermistor data and the "soliton-like" signature of acoustic data is difficult to support due to the significant differences between the sampling rate of hydrographic data (1 sample every 10 minutes) and the rate of acoustic transmissions ( 1 transmission every 8 seconds). To clarify this issue we exploited the theoretical knowledge on soliton propagation to generate "soliton-like" fields of temperature and sound velocity and used them as input for the acoustic model C-SNAP (Ferla et al., 1993). From a previous estimation on internal tide propagation (Rodríguez et al., 1998) the angle of propagation of SWP was estimated as $\theta \approx 15^{\circ}$ (see Fig.10). 


\section{O.C. RODRÍGUEZ ET AL.}

The solutions Eqs.(2) and (4) were used to derive a single "sech" profile and several "dnoidal" profiles, with the number of solitons within each packet being controlled by $\mathrm{S}$ and $\tau$. Acoustic simulations were performed with $\mathrm{C}$ SNAP at $430 \mathrm{~Hz}$ and for each of such fields, as if SWPs were propagating from the VLA to the North Position. For each soliton profile it was found a complex dependence of TL on the particular characteristics and position of the SWP. The best result of simulations was obtained for a "dnoidal" packet with four solitons (see Fig.11). Simulated TL reproduces the behaviour of attenuation, signal increase and attenuation again. However the pattern is not symmetric around the attenuation maximae and the signal increase lasts twice longer than observed. Additional calculations of TL at more frequencies (see Fig.12) reveal the effect of signal increase if the constraint of keeping only 10 propagating modes ${ }^{1}$ is applied. Again in simulations the effect of signal increase is broader than that observed, and it holds only for some frequencies.

The simulations described above concerned propagation from the point of view of normal modes. However, it was also important to obtain some support with the help of ray tracing. In order to accomplish this task it was performed a preliminary range-independent ray tracing for a narrow beam of rays (see Fig.13). From that figure it can be seen that the beam width increases monotonically with range. Two cases of range-dependent ray tracing were considered: the first for the position of the "dnoidal" SWP where signal attenuation was found (see Fig.14, on top), and the second, where the SWP leads to signal increase (see Fig.14, on bottom). From the ray tracing it can be seen that the SWP leads to additional refraction of rays at the end of the ray beam. However, the refraction acts in different ways depending on the position of the SWP: when compared with the rangeindependent case, the beam becomes wider in the first case and narrower (or "focussed") in the second. This indicates that one can expect a decrease of signal amplitude followed by its increase as the SWP propagates towards the source. These ray tracing simulations agree qualitatively with the results that were obtained with C-SNAP.

The quantitative differences between real data and simulations indicate that the soliton profile which provided the best results does not constitutes the closest estimate to the real soliton perturbation. However, the qualitative agreement achieved confirms the assumption of propagating SWPs providing the appropriate conditions for the observation of the signal increase.

\footnotetext{
${ }^{1}$ This is twice more than the number of propagating modes considered in (Zhou et al., 1991).
} 


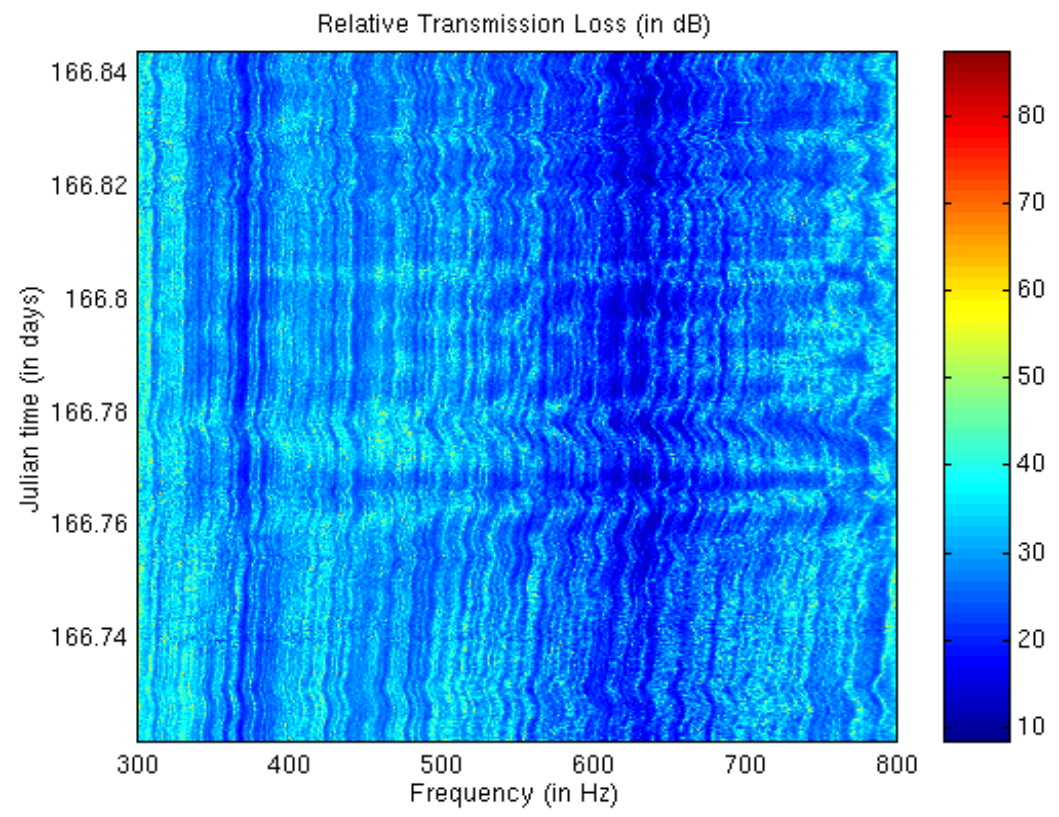

Figure 8. Relative transmission loss in $\mathrm{dB}$ along frequency.

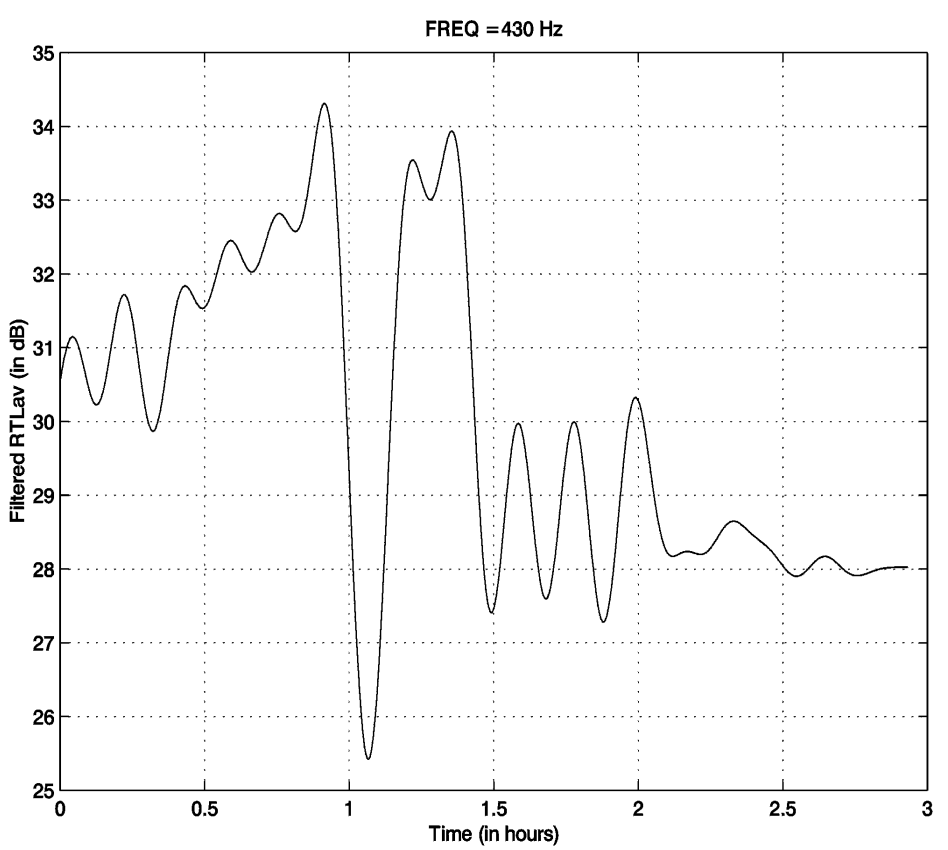

Figure 9. Averaged and smoothed transmission loss at $430 \mathrm{~Hz}$. 
O.C. RODRÍGUEZ ET AL.

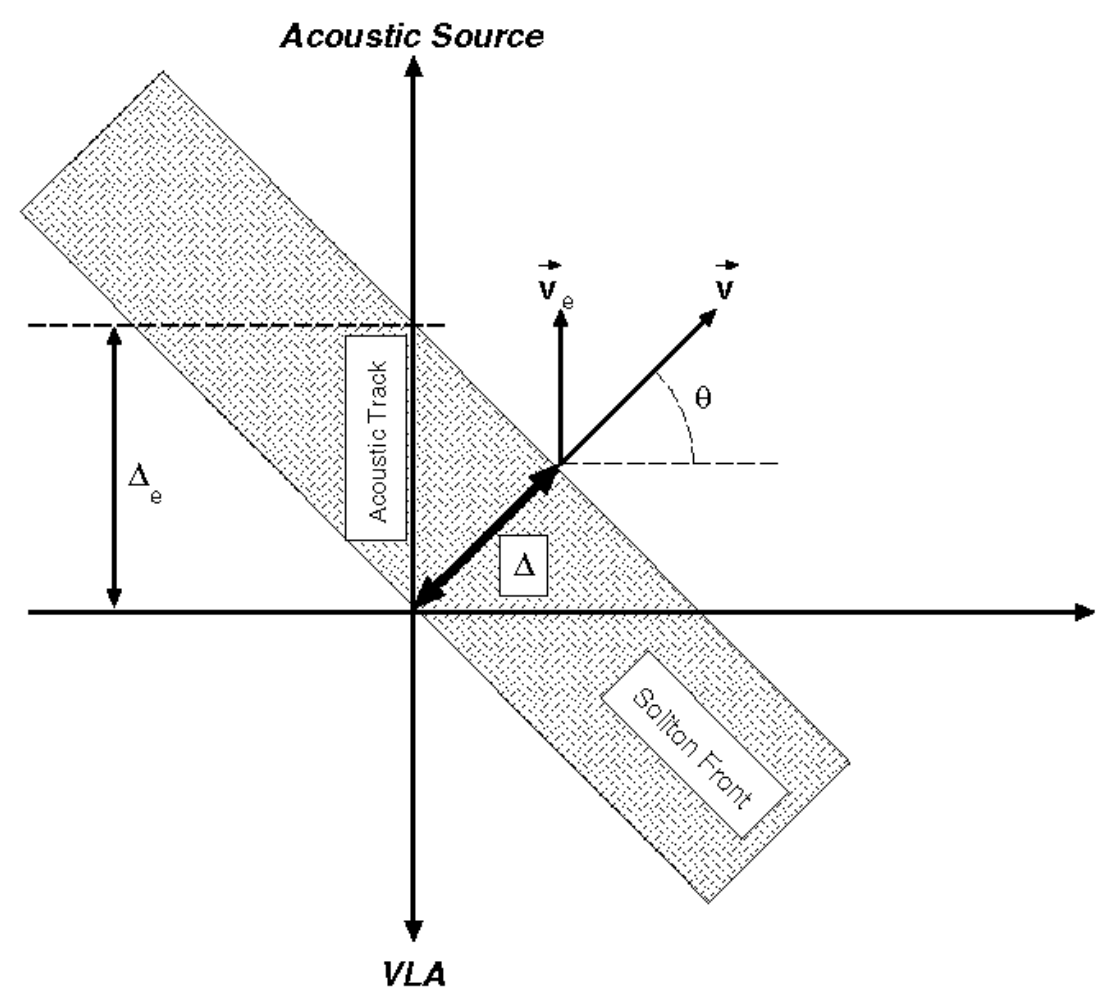

Figure 10. Considered geometry of SWP propagation. 


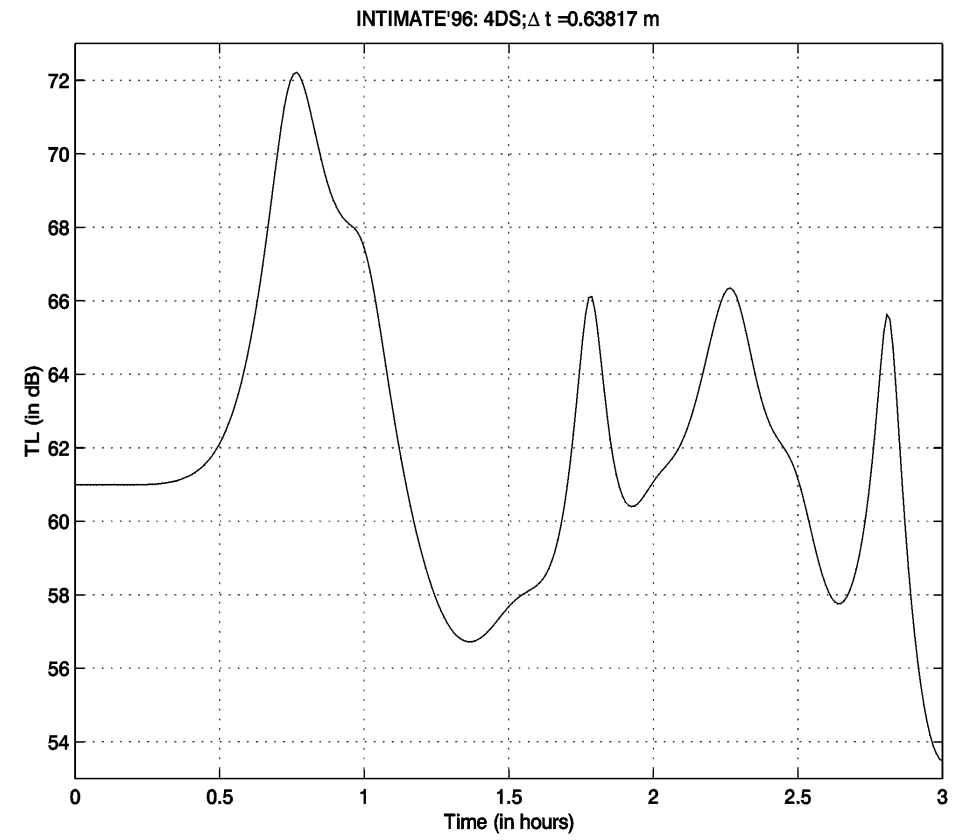

Figure 11. Simulated transmission loss at $430 \mathrm{~Hz}$.

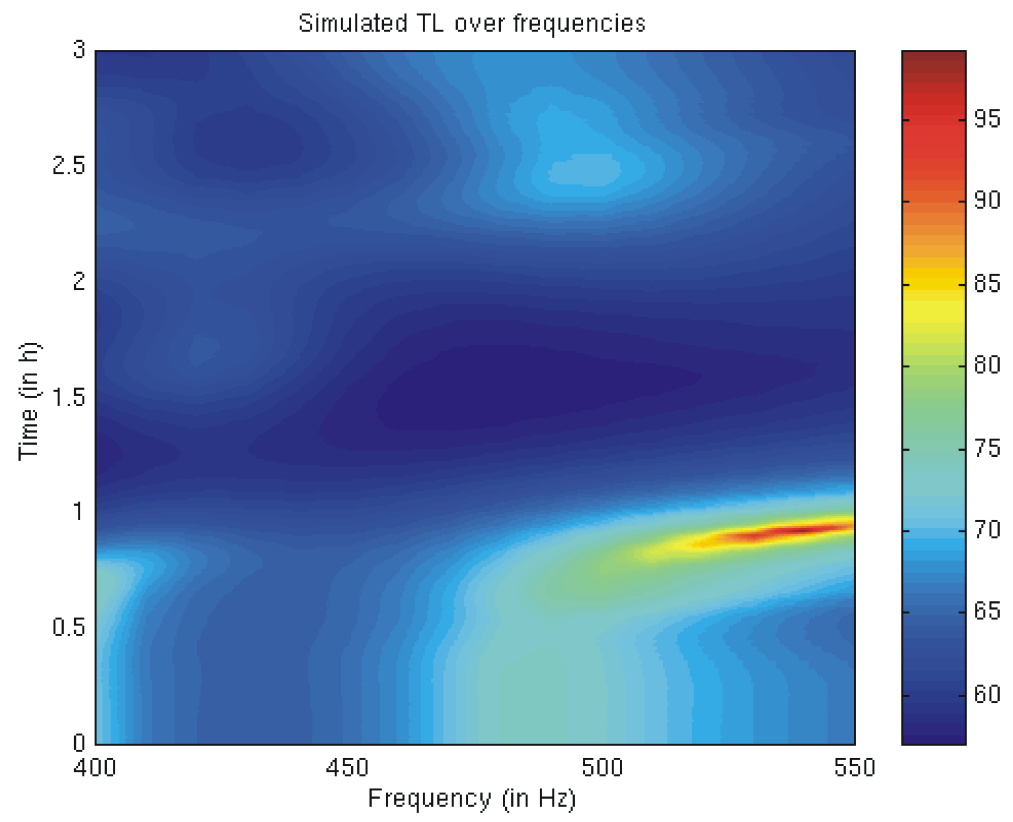

Figure 12. Dynamic simulations over frequency. 
O.C. RODRÍGUEZ ET AL.

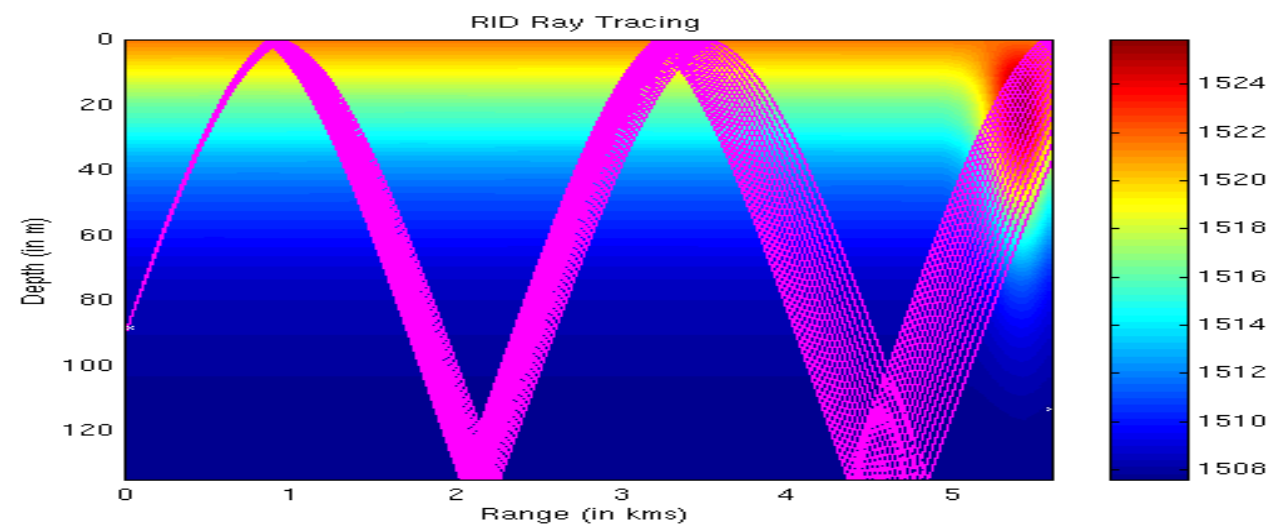

Figure 13. Range-independent ray-tracing; part of the "dnoidal" SWP is plotted for comparison with the range-dependent cases (see below).
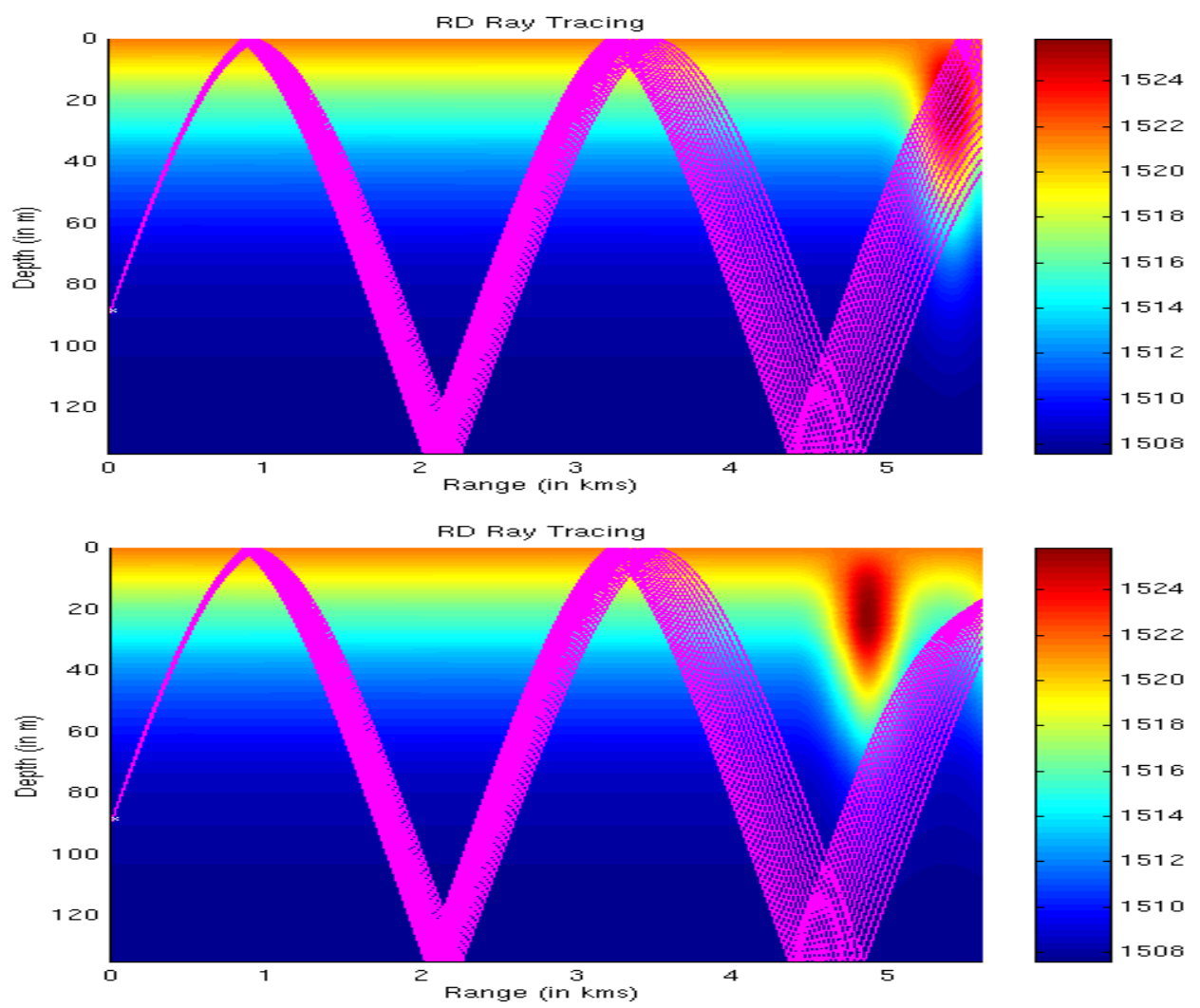

Figure 14. Range-dependent Ray-tracing showing beam spreading (on top) and focussing (on bottom). 


\section{Conclusions}

On the basis of this analysis the following conclusions can be drawn: based on the nonlinear rotationless form of Hydrodynamic Equations it can be shown that a stratified environment admits the generation of "modal" solitons, with characteristics that depend on Hydrodynamic Normal Modes (HNMs); the "sech" solution of the KdV equation describes a single modal nonlinear perturbation, which propagates in range and time without changing its shape; the "dnoidal" modal solution of the same equation describes a dynamically evolving SWP, where the number of solitons inside the packet varies in range and time; further analysis of the nonlinear rotationless form of Hydrodynamic Equations indicates that modal amplitudes of current and temperature will be proportional to modal vertical displacement, i.e., will exhibit a similar "soliton-like" shape; this can be exploited to generate physically consistent "soliton-like" distributions of temperature and sound velocity; filtering of modal amplitudes of current and thermistor records from the INTIMATE'96 experiment into low-pass and high-pass frequency components reveals possible propagation of SWPs across the experimental site; one of the SWPs is coincident with an increase of signal amplitude, which can be due to a focussing effect; acoustic range-dependent and timedependent simulations through "soliton-like" fields of sound velocity agree with observations when a particular "dnoidal" profile is considered; the results of normal-mode calculations are qualitatively supported with rangedependent ray tracing tests for the positions of the SWP where the normal mode results indicate successive signal attenuation and signal increase. 


\section{O.C. RODRÍGUEZ ET AL.}

\section{References}

Small, J. and Hallock, Z. and Pavey, G. and Scott J. (1995), Observations of large amplitude internal waves at the Malin Shelf edge during SESAME 1995, Continental Shelf Research

Jeans, D.R.G. and Sherwin, T.J. (1996) Solitary Internal Waves on the Iberian Shelf, MORENA Scientific and Technical Report No.44 Project MAST II MAS2-CT930065

Zhou, J., Zhang, X. and Rogers, P. (1991) Resonant interaction of sound wave with internal solitons in the coastal zone, J. Acoust. Soc. America Vol. 90 no. 4, pp. 20422054

Caille, G.W., Dahl, P.H., Gan, Z., Jin, G., Lei, L., Rogers, P.H., Spindel, R.C., Sun, Z. Zhang, R. and Zhou J. (1997) Overview of the Joint Chine-U.S. Yellow Sea-96 Experiment, Proceedings of the SWAC'97 Conference in Beijing pp. 17-22

Apel J.R.(1987), Principles of Ocean Physics

Ostrovsky, L.A. (1978) Nonlinear Internal Waves in a rotating ocean, Oceanology Vol. 18 no. 2, pp. 181-191, (in russian)

Apel, J.R., Badiey, M., Chiu, C., Finette, S. , Headrick, R., Kemp, J., Lynch, J.F., Newhall, A., Orr, H., Pasewark, B.H., Tielburger, D., Turgut, A., Heydt, K. and Wolf, S. (1997) An Overview of the 1995 SWARM Shallow-Water Internal Wave Acoustic Scattering Experiment, IEEE Journal of Oceanic Engineering Vol. 22 no. 3, pp. $465-500$

Gabov S.A. (1988) Introduction to the theory of nonlinear waves (in russian)

Apel, J., Orr, M., Finette, S. and Lynch, J. (1997) A new model for internal solitons and tides on the continental shelf, Proceedings of the SWAC'97 Conference in Beijing pp. 219-225

LeBlond, P.H. and Mysak, L.A. (1989) Waves in the Ocean

Rodríguez, O.C., Jesus, S., Stephan, Y., Demoulin, X., Porter, M. and Coelho, E. (1998) Internal Tide Acoustic Tomography: Reliability of the normal modes expansion as a possible basis for solving the inverse problem, Proceedings of the 4 th. European Conference on Underwater Acoustics in Rome pp. 587-592

Ferla, C.M., Porter, M.B. and Jensen, F.B. (1993) C-SNAP: Coupled SACLANTCEN normal mode propagation loss model, SACLANT UNDERSEA RESEARCH CENTRE (SM-274), La Spezia, Italy 